\title{
Simultaneous time resolved X-ray scattering experiments in the small and wide angle region
}

\author{
W. BRAS ${ }^{* * *}$, G.E. DERBYSHIRE ${ }^{* *}$, A.J. RYAN ${ }^{* * *}$, G.R. MANT ${ }^{* *}$, P. MANNING ${ }^{* *}$, \\ R.E. CAMERON ${ }^{* * * *}$ and W. MORMANN ${ }^{* * *}$ \\ ${ }^{*}$ Netherlands Organisation for Scientific Research (NWO) \\ ** SERC Daresbury Laboratory, Warrington WA4 4AD, U.K. \\ *** University of Manchester, Institute of Science and Technology, U.K. \\ ${ }^{* * * *}$ Cavendish Laboratory, Cambridge, U.K. \\ ***** Universität-GH Siegen, Germany
}

\begin{abstract}
A SAXS synchrotron beam line is equipped with a second detector system so that simultaneous WAXS experiments can be performed. Modular sample environment cells give the possibility to obtain extra thermodynamical information. Experiments have been performed on a variety of samples.
\end{abstract}

\section{Introduction}

There is a wide variety of experiments in which it is essential to obtain structural information that spans the real space dimension range from $1-1000 \AA$. Polymers, liquid crystals and membranes are a few important examples. If one wants to study phase transformations in these systems it is crucial to collect the data in a time resolved mode.

A problem with performing independent time resolved Wide and Small Angle X-ray scattering experiments on a sample is to obtain a proper time correlation between the two experiments. When a third independent experiment, like Differential Scanning Calorimetry or Fourier Transform Infrared spectroscopy, has to be time correlated to the scattering experiments as well the situation is aggravated. Equipment in which these experiments can be combined would offer advantages for radiation sensitive biological samples as well.

Several attempts to build equipment that would allow simultaneous scattering experiments have been described in the literature $[1,2,3]$. Most of this equipment has some limitations in either the q-range that can be observed or in the obtainable time resolution.

A major problem in performing these experiments is that, in order to obtain a large q-range, one generally has to use position sensitive delay line detectors since solid state detectors in general are too short. For WAXS experiments this introduces a serious problem due to the parallax problem that these detectors exhibit when $X$-ray impinge on a large angle with respect to the surface normal of the detector.

Recently we have assembled equipment that could be utilised for the above described experiments with a larger obtainable q-range and a higher time resolution [4]. Here we describe the improvements to the initial equipment and show some results from the diverse set of recently performed experiments.

\section{Description equipment}

The optical system of station 8.2 at the Synchrotron Radiation source in Daresbury, which forms the basis of the equipment, has been described elsewhere in more detail [4]. A triangular bent $\mathrm{Ge}(111)$ crystal is used to focus the $\mathrm{X}$-rays in the horizontal plane and monochromate at $1.51 \AA$. Subsequent vertical focusing is obtained by using an uncoated quartz mirror under grazing incidence. The total flux on the sample is $5 \times 10^{10}$ photons $/ \mathrm{sec}$ in a $3.5 \times 0.3 \mathrm{~mm}^{2}$ spot. The horizontal and vertical divergence are, respectively, 5.0 and $0.25 \mathrm{mrad}$. The distance between the last two slit sets is increased by 1.0 meter with respect to the conventional set-up in order to reduce the parasitic scatter opening angle. 


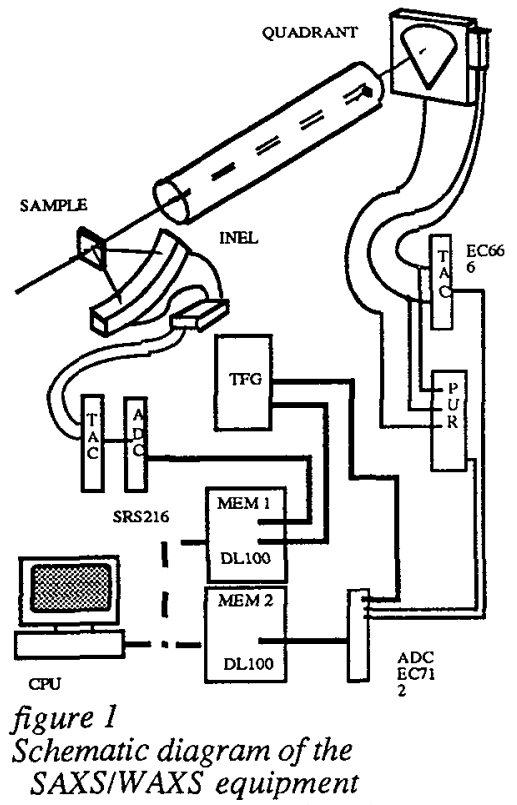

A schematic diagram of the equipment used in our experiments is shown in figure 1 . The SAXS pattern above the direct beam is recorded with a standard Daresbury quadrant detector [5]. The WAXS pattern below the direct beam is collected with a commercially available curved INEL detector that is positioned with the centre of its radius of curvature at the sample position. By changing the sample to quadrant detector distance it is possible to obtain overlap between the two data regions. A vacuum chamber is constructed that covers both scattering regimes.

The sample stage is multi-functional. Routinely the sample temperature can be controlled between $-196^{\circ}$ and $+600^{\circ} \mathrm{C}$. The sample housing can be evacuated or filled with inert gasses. Thanks to its modular design it is possible to mount more specialised equipment such as high temperature stages $\left(>600^{\circ} \mathrm{C}\right)$, DSC heads, FTIR probes or temperature quench equipment.

\section{Results}

In figure 2 a SAXS calibration pattern from a wet collagen specimen is shown. The observable q-range is from 0.0079 to $0.2 \AA^{-1}$. Figure 3 shows the diffraction pattern from the NBS silicon powder diffraction calibration standard. The data range is from $q=0.41$ to $5.7 \AA^{-1}$. The gap in the data range can be closed completely if one is prepared to sacrifice a small part of the lowest angle region.

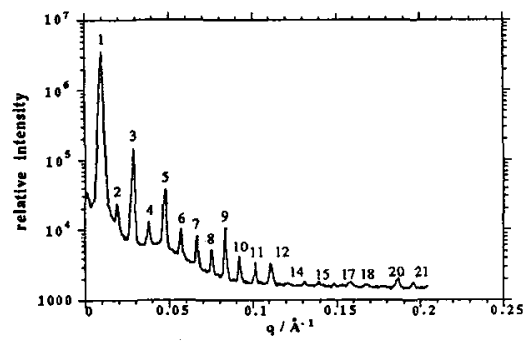

Figure 2

Wet rat tail collagen spectrum (670 A repeat distance)

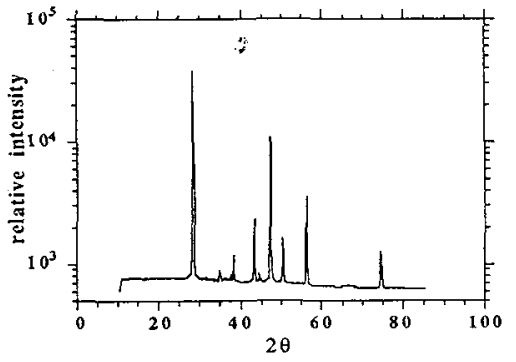

Figure 3

NBS silicon powder diffraction standard

The fact that the detector is focused on neither detector might be a reason for concern about the spatial resolution obtainable. The SAXS pattern, however, is excellent in its spatial resolution. The WAXS 
data quality is superior to a standard $\theta / 2 \theta$ diffractometer using filtered $C u K \alpha$ radiation. This can not be explained by a difference in bandpass for the radiation, since $\Delta \lambda / \lambda$ is roughly equal for both situations. The explanation can be found in the lower divergence of the synchrotron beam line.

The electronic system time resolution is $10 \mu \mathrm{s}$, but the practical time resolution that can be obtained with this equipment is approximately $100 \mathrm{~ms}$. This limitation is imposed due to the limited count rate that the INEL can handle. Generally the scattering pattern has to be attenuated by a factor $30-300$ times in order not to exceed the count rate limits of this detector. The quadrant detector only has to be attenuated by a factor $3-10$.

\section{Examples}

Successful experiments have been performed on a variety of samples with an emphasis on material science research but also on some biological materials. Examples of the material science program are the study of the annealing behaviour of cordierite glasses, the freezing of undercooled water and a wide variety of temperature induced phase transitions in polymer systems. The growth process of starch granules, the temperature induced rearranging of lipid skin and artificial membranes and changes in structure of bovine cornea upon hydration are examples of the biological program. Below three example of experiments are shown.
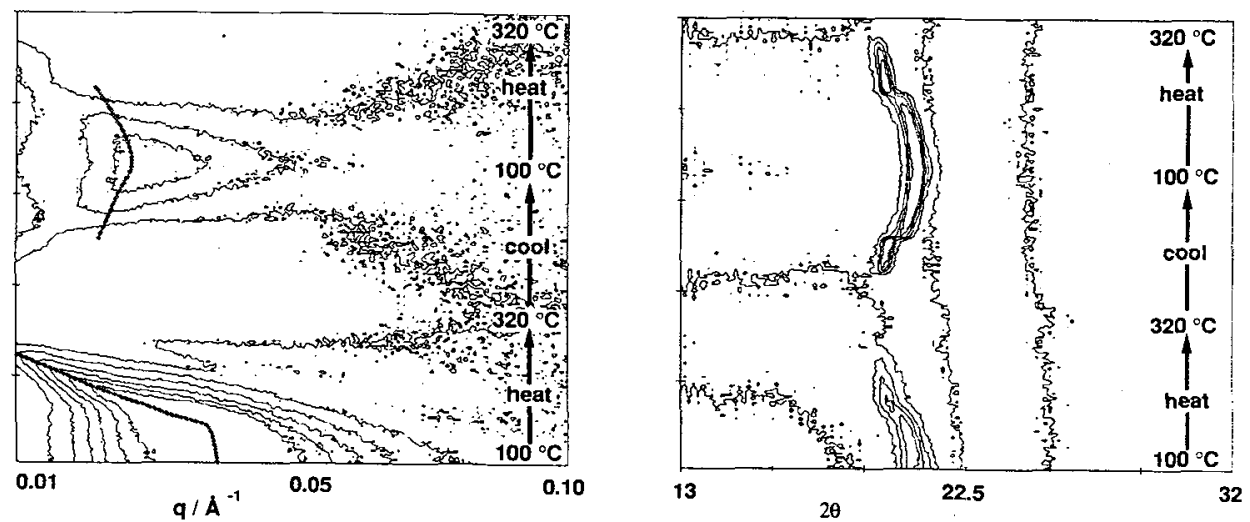

Figure 4 Contour plots of time resolved experiment on a block copolyester sample.

The data in figure 4 is obtained during a heat $\rightarrow \mathrm{coOl} \rightarrow$ heat cycle on a liquid crystal block copolyester. The SAXS and WAXS data are two intensity contour in $\mathrm{Iq}^{2}$ and I respectively. The phase transitions from crystal 1 to crystal 2 are readily apparent. The sample was heated with $20^{\circ} \mathrm{C} / \mathrm{min}$. The data was collected in frames of 10 seconds.

The second example concerns the growth of starch granules (see figure 5). Here the gelatinization process under the influence from a temperature increase from $40^{\circ}$ to $80^{\circ} \mathrm{C}$ was studied. The loss of crystallinity, as evidenced by the decrease in intensity of the WAXS peaks, is associated with the dissociation of the amylopectin double helices. The single peak in the SAXS region exhibits a similar loss in intensity due to the disruption of the lamellar structure as water penetrates the granule. 


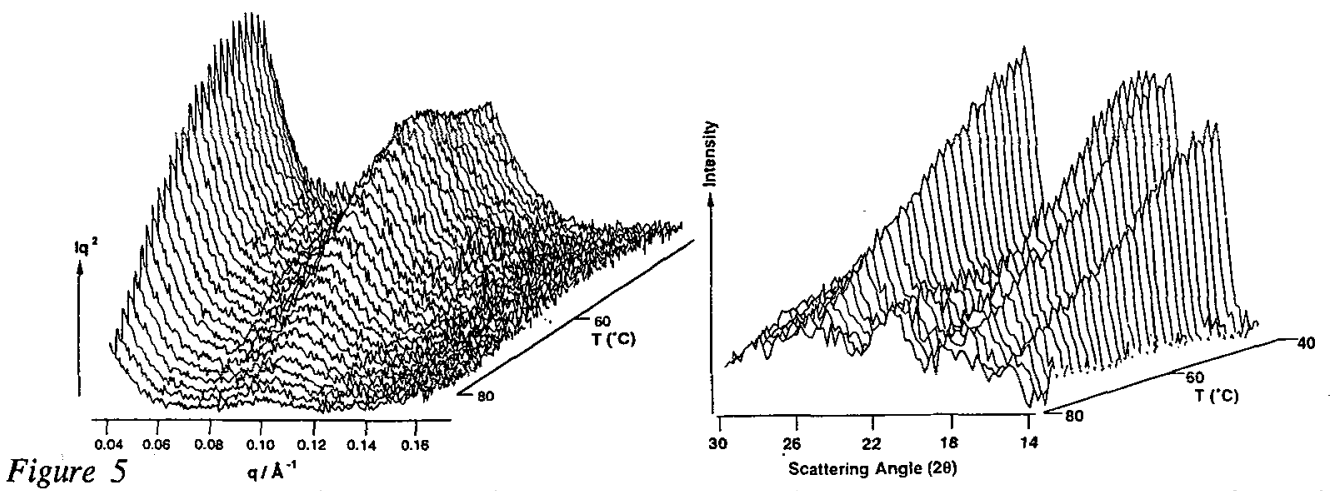

Gelatinanization of $57 \%$ wheat starch under the influence of a temperature increase from $40^{\circ}$ to $80^{\circ} \mathrm{C}$

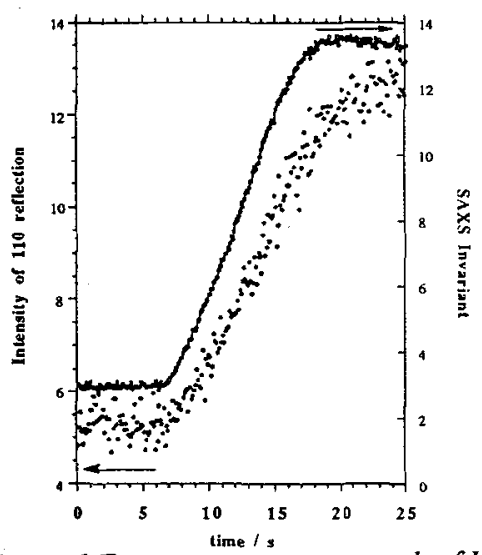

Data obtained from a fast temperature quench experiment on High Density Polyethylene. The sample was quenched from the molten state with $\approx 10^{\circ} \mathrm{C} / \mathrm{s}$. Time resolution $0.1 \mathrm{~s}$. The SAXS invariant is shown (right scale) and the intensity of the 110 reflection (left scale), which can be used as a measure of the degree of crystallinity. This experiment indicates that it is feasible to work at a high time resolution, making this instrument very useful in the study of fast phase transitions.

Figure 6 Fast temperature quench of HDPE

\section{Conclusion}

The equipment described here is a very versatile instrument especially suited to the real time study of phase transitions. A time resolution of up to $0.1 \mathrm{~s}$ and a very wide scattering range can be studied. The combination of SAXS, WAXS and DSC will be tried out in the near future. Attempts to combine FTIR experiments with the scattering techniques will also be undertaken .

We would like to thank S.Benadda and P. Jenkins for their assistance in the sample preparation. Also we would like to express our appreciation for the support we have received from our colleagues and the assistance from the technical staff at Daresbury Laboratory.

\section{Literature}

[1] P.Laggner and H.Mio, NIMPR, A323,(1992)86-90

[2] M.Bark, C.Schulze and H.G.Zachman

Polym.Prep.Am.Chem.Soc.Div.Polym.Chem.(1990),31(2), 106-107

[3] K.Tashiro, M.M.Satkowski, R.S.Stein, Y.Li, B.Chu, S.L.Hsu.

Macromolecules (1992), 25, 1809 - 1815

[4] W.Bras, G.E.Derbyshire, A.J.Ryan, G.R.Mant, A.Felton, R.A.Lewis, C.J.Hall, G.N.Greaves. NIMPR A (1993),326,587-591

[5] R.A.Lewis, I.Sumner, A.Berry, J.Bordas, A.Gabriel, G.Mant, B.Parker, K.Roberts, J.Worgan. NIMPR A (1988),273,773-777 